Densidad y patrones de actividad del

ocelote en la selva Lacandona

\title{
Density and activity patterns of ocelots in the Greater Lacandona Ecosystem
}

\author{
J. Antonio de la Torre ${ }^{1 *}$, Paulina Arroyo-Gerala ${ }^{1}$, and Leonora Torres-Knoop ${ }^{2}$
}

\begin{abstract}
1 Instituto de Ecología, UNAM, Laboratorio de Ecología y Conservación de Vertebrados Terrestres, Coyoacán, 04510. Ciudad de México, México. Email: adelatorre@iecologia.unam.mx (JAT), paw.ar.ger@hotmail.com (PA-G).

2 Intituto de Biología UNAM, Laboratorio de Análisis Espaciales, Circuito Exterior s/n, Ciudad Universitaria, Coyoacán, 04510. Ciudad de México, México. Email: Itorresknoop@gmail.com (LT-K).

* Corresponding author
\end{abstract}

The ocelot is the third largest cat that occurs in Mexico. Despite all the information that has been collected regarding its distribution in the country, little is known about its basic ecology in Mexico. In this study we estimated population density of ocelots and their activity patterns for the Greater Lacandona Ecosystem region. Data were obtained through camera traps. We used 33 camera trap stations that were active during 60 consecutive days. The effective sampling effort was 1,920 trap days. Population density was estimated through Bayesian Spatial Explicit Capture-Recapture Models. In addition, the activity pattern of ocelots was determined by the time recorded of all photographs obtained. We estimated a minimal density of $12.9 \pm 5.39$ ocelots for each $100 \mathrm{~km}^{2}$ for the southern Montes Azules Biosphere Reserve. According with the photographic records we identified that ocelots were active both day and night. Our results are similar to those obtained in other areas located in the tropical rain forests of Central America with the classic capture-recapture methods. In addition, this study shows the potential of this preserved area for maintaining long-term population of ocelots in the tropical rain forest of the Greater Lacandona Ecosystem. More information about this species needs to be obtained, especially for regions characterized by habitat fragmentation and human perturbation.

El ocelote es el tercer felino más grande que habita en México. A pesar de que recientemente se ha publicado mucha información acerca de la distribución de esta especie en el país, aún se desconoce muchos aspectos de su ecología básica en México. Con la finalidad de contribuir en el conocimiento de la ecología poblacional de esta especie en las zonas tropicales de México, en este estudio reportamos la densidad del ocelote para la región de la Selva Lacandona, así como sus patrones actividad. El estudio se hizo mediante trampas-cámara. Utilizamos 33 estaciones de foto-trampeo que estuvieron activas durante 60 días consecutivos, teniendo un esfuerzo efectivo de muestreo de 1,920 días-trampa. El análisis de densidad se hizo a partir de modelos espacialmente explícitos bayesianos. El patrón de actividad de los ocelotes se determinó por medio de la hora que registraron todas las fotografías que se obtuvieron de la especie. Estimamos una densidad mínima de $12.9 \pm 5.39$ SE ocelotes $/ 100 \mathrm{~km}^{2}$ para el sur de la Reserva de la Biosfera Montes Azules. De acuerdo con las fotografías obtenidas, los ocelotes mostraron picos de actividad tanto en el día como en la noche. Estos resultados son similares a otras densidades poblacionales reportadas para esta especie con métodos de captura-recaptura clásicos para los bosques tropicales húmedos de Centroamérica. El presente estudio ilustra el el potencial de las zonas conservadas de la región de la Selva Lacandona para mantener una población de ocelotes viable a largo plazo. Hace falta ampliar el conocimiento de la especie, especialmente en regiones caracterizadas por la fragmentación y la perturbación humana.

Key words: abundance; activity; camera traps; Leopardus pardalis; SPACECAP.

๑ 2016 Asociación Mexicana de Mastozoología, www.mastozoologiamexicana.org 


\section{Introducción}

El ocelote (Leopardus pardalis) es una de las once especies de felinos que habitan en el Neotrópico y el tercer felino más grande que habita en México. Su distribución abarca desde el sur de Estados Unidos hasta el norte de Argentina y ocupa una gran diversidad de ecosistemas como bosques tropicales, pastizales y matorrales (Sunquist y Sunquist 2009). En México, el ocelote se puede encontrar tanto en regiones tropicales como subtropicales y su distribución incluye en el norte los estados de Sonora y Tamaulipas, extendiéndose hacia el sur a lo largo de las vertientes costeras del Golfo de México y del Pacifico hasta Chiapas y Yucatán. En el centro del país se distribuye a través de la cuenca del río Balsas en el Estado de México (Leopold 1959; Hall 1981). Incluso, existen registros recientes de esta especie en los estados de Zacatecas y Aguascalientes (Bárcenas y Medellín 2010; Valdez-Jiménez et al. 2013).

La cacería de los ocelotes por sus pieles en la década de los 1960 y 1970 s redujo de manera considerable muchas de las poblaciones de esta especie en toda su área de distribución (Nowell y Jackson 1996; Di Bitetti et al. 2008; Sunquist y Sunquist 2009). Como consecuencia de la amenaza que esto representaba, el ocelote fue catalogado como Vulnerable en la lista roja de la Unión Internacional para la Conservación de la Naturaleza (UICN). Sin embargo, tras la prohibición del comercio de pieles de felinos silvestres en 1980 por la Convención sobre el Comercio Internacional de Especies Amenazadas de Flora y Fauna (CITES por sus siglas en inglés), la categoría de conservación de esta especie cambió a Preocupación Menor (Paviolo et al. 2015). En México, el ocelote se encuentra clasificado como "en Peligro de Extinción" (SEMARNAT 2010). Sin embargo, aún falta mucha información sobre aspectos básicos de la ecología de esta especie en México, la cual es necesaria para diseñar los programas de manejo y conservación adecuados para esta especie en el país.

La mayor parte de la información publicada sobre el ocelote en México consiste en registros de su distribución (Pozo de la Tijera y Escobedo Cabrera 1999; Grigione et al. 2001; Sanchez et al. 2002; Servín et al. 2003; Lorenzana et al. 2004; Iglesias et al. 2008; Bárcenas y Medellín 2010; Ramirez-Bravo et al. 2010; Martínez-Calderas et al. 2011; Aranda et al. 2012; Ahumada-Carrillo et al. 2013; Hernández-Flores et al. 2013; Sánchez Hernández et al. 2013; Galindo-Aguilar et al. 2016; García-Bastida et al. 2016). Aunque también hay algunos estudios sobre su historia natural los cuales han contribuido al conocimiento de sus hábitos alimenticios (de Villa et al. 1995), y patrones de actividad (Pérez-Irineo y Santos-Moreno 2014; Briones-Salas et al. 2016). A pesar de que se han publicado varios estudios acerca de la densidad de esta especie a lo largo de su intervalo de distribución (Trolle y Kéry 2003; 2005; Maffei et al. 2005; Di Bitetti et al. 2006; 2008; Dillon y Kelly 2007; Salvador y Espinosa 2015), se tiene muy poca información acerca de las densidades de esta especie para México (Pérez-Irineo y Santos-Moreno 2014; Martínez-Hernández et al. 2015). El objetivo de este estudio es estimar la densidad poblacional de los ocelotes en la región de la Selva Lacandona, en el estado de Chiapas, a partir del uso de trampas-cámara y de modelos espacialmente explícitos de captura-recaptura. Adicionalmente, describimos los patrones de actividad de los ocelotes en diferentes periodos del día utilizando los registros obtenidos mediante las trampas-cámara y comparamos los resultados obtenidos con otros estudios publicados para México y otras regiones del Neotrópico.

\section{Material y Metodos}

Área de estudio. La Selva Lacandona se ubica en el sureste de México. Esta región contiene la porción continua más grande de bosque tropical lluvioso de México y consiste en un área prioritaria para la conservación. Esta región es la más rica del país en términos de diversidad y alberga muchas especies catalogadas como amenazadas y en peligro de extinción (Medellín 
1994). Se ha estimado que esta región contiene más de 4,000 especies de plantas vasculares y alberga entre $20 \%$ y $25 \%$ de la diversidad de especies de plantas y animales de México (Medellín 1994; Davis et al. 1997).

La Selva Lacandona representa una de las regiones más amenazadas del país. De las 1,500,000 hectáreas originales de selva, se han perdido dos terceras partes en los últimos 40 años (Mendoza y Dirzo 1999; Jong et al. 2000). Las principales amenazas para este ecosistema son el rápido crecimiento demográfico, las altas tasas de deforestación y la extracción no regulada de recursos no renovables (Medellín 1994). La mayor parte del remanente de bosque de esta región se encuentra protegida a través de siete áreas naturales: la Reserva de la Biosfera Montes Azules (RBMA, 331,200 ha), la Reserva de la Biosfera Lacan-Tun (61,873 ha), el Monumento Natural Bonampak (4,757 ha), el Monumento Natural Yaxchilán (2,621 ha), el Área de Protección de Flora y Fauna Chan-Kin (12,184 ha), el Área de Protección de Flora y Fauna Nahá (3,847 ha) y el Área de Protección de Flora y Fauna Metzabok (3,368 ha).

El área de muestreo se localiza en el sur de la Selva Lacandona, en el borde sur de la Reserva de la Biosfera Montes Azules, dentro de la zona de uso restringido de la reserva (INE-SEMARNAP 2000). Se encuentra aislada de los ejidos colindantes (Loma Bonita, Boca de Chajul, Playón de la Gloria y Galacia) por el río Lacantún (Figura 1). La vegetación dominante en este sitio es selva alta perennifolia bien conservada (de la Torre y Medellín 2011).

Diseño de muestreo. El sitio de estudio comprendió un área de $82 \mathrm{~km}^{2}$, calculada a través del método de polígono mínimo convexo y considerando el área cubierta por las trampas-cámara que utilizamos para este estudio (Figura 2). Tomamos registros durante 60 días consecutivos,

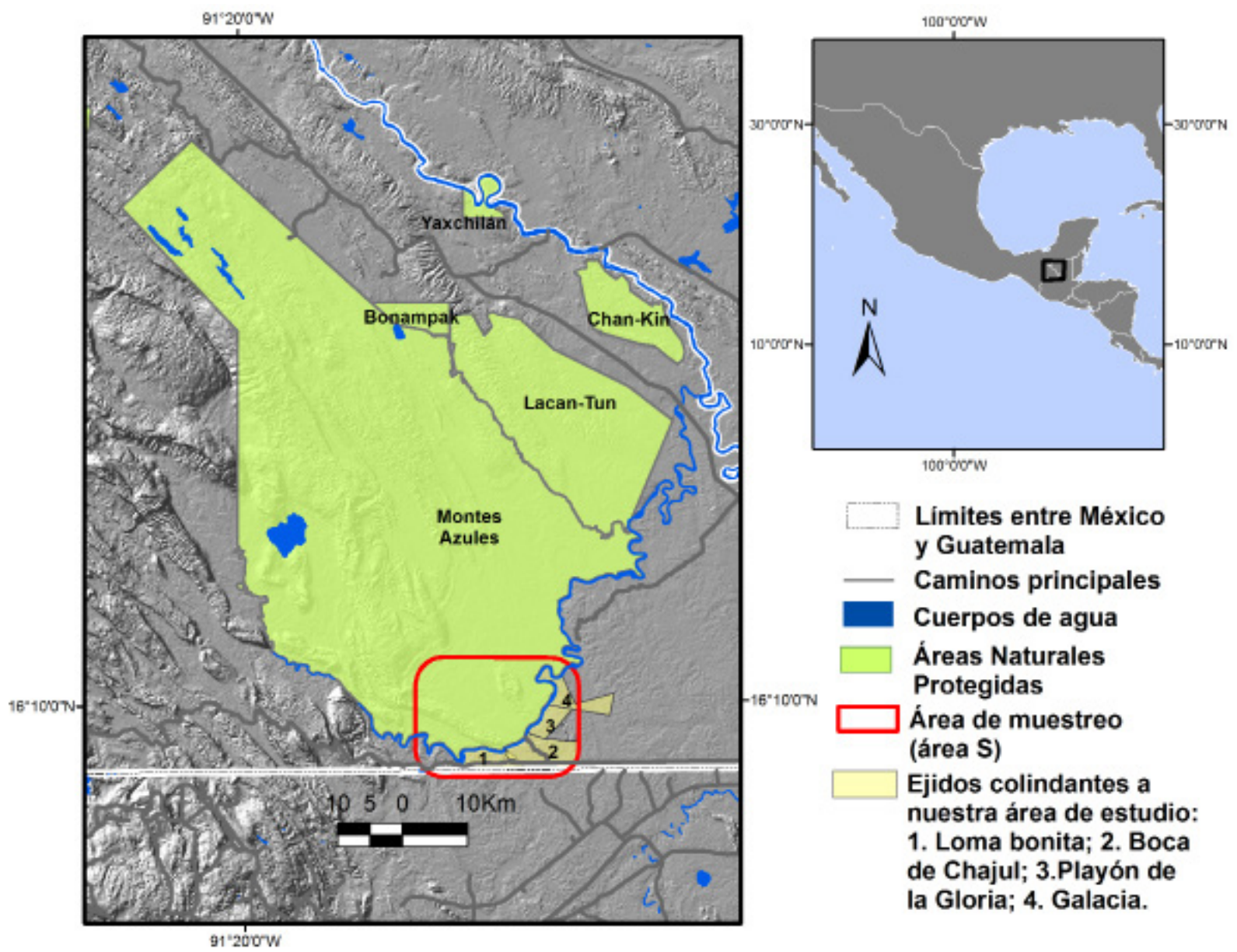

Figura 1. Localización del área de estudio en el paisaje de la Selva Lacandona. 
a partir de noviembre del 2007 hasta enero del 2008. Colocamos un total de 33 estaciones de muestreo, de las cuales 17 consistieron en estaciones dobles, es decir, colocamos dos trampascámara viéndose entre sí con el fin de capturar ambos perfiles de los individuos (Figura 2). Se definió como un evento de muestreo cada día que estuvieron activas las estaciones de fototrampeo (cada 24 horas = un evento de muestreo) y como captura a una fotografía de un individuo de ocelote diferente dentro de cada evento de muestreo. Dado que una de las trampas cámara de una de las estaciones sencillas fue robada durante el muestreo, en total logramos un tiempo efectivo de muestreo de 1920 días-trampa.

El equipo consistió en 29 cámaras Camtrakker ${ }^{\circledast}$ (Camtrakker TM, Camtrack South Inc. Georgia, EE. UU.) y 21 Deer Cam ${ }^{\circledast}$ (modelo DC-200: Non Typical Inc., Park Lane, Park Falls, EE. UU). Las trampas cámara se colocaron aproximadamente a $45 \mathrm{~cm}$ del suelo y con una distancia de al menos tres metros de los senderos. Dado que el protocolo utilizado en este estudio estaba enfocado a la estimación de abundancias de los jaguares (de la Torre y Medellín 2011), la separación entre las estaciones de muestreo fue de 1 a $3 \mathrm{~km}$ y éstas se colocaron en sitios asociados a rastros de jaguares o que se consideraron familiares a ellos. Sin embargo, durante el muestreo se obtuvieron una gran cantidad de registros de muchas otras especies además de los jaguares, incluyendo los ocelotes (de la Torre 2009).

Estimación de la densidad poblacional. De acuerdo al patrón de manchas individual de cada uno de los ocelotes, identificamos el mayor número de individuos posible (Trolle y Kéry 2003; Maffei et al. 2005; Di Bitetti et al. 2006; Dillon y Kelly 2007). Sin embargo, dado que obtuvimos pocas fotografías de ambos perfiles de los individuos, separamos el número de individuos fotografiados del perfil

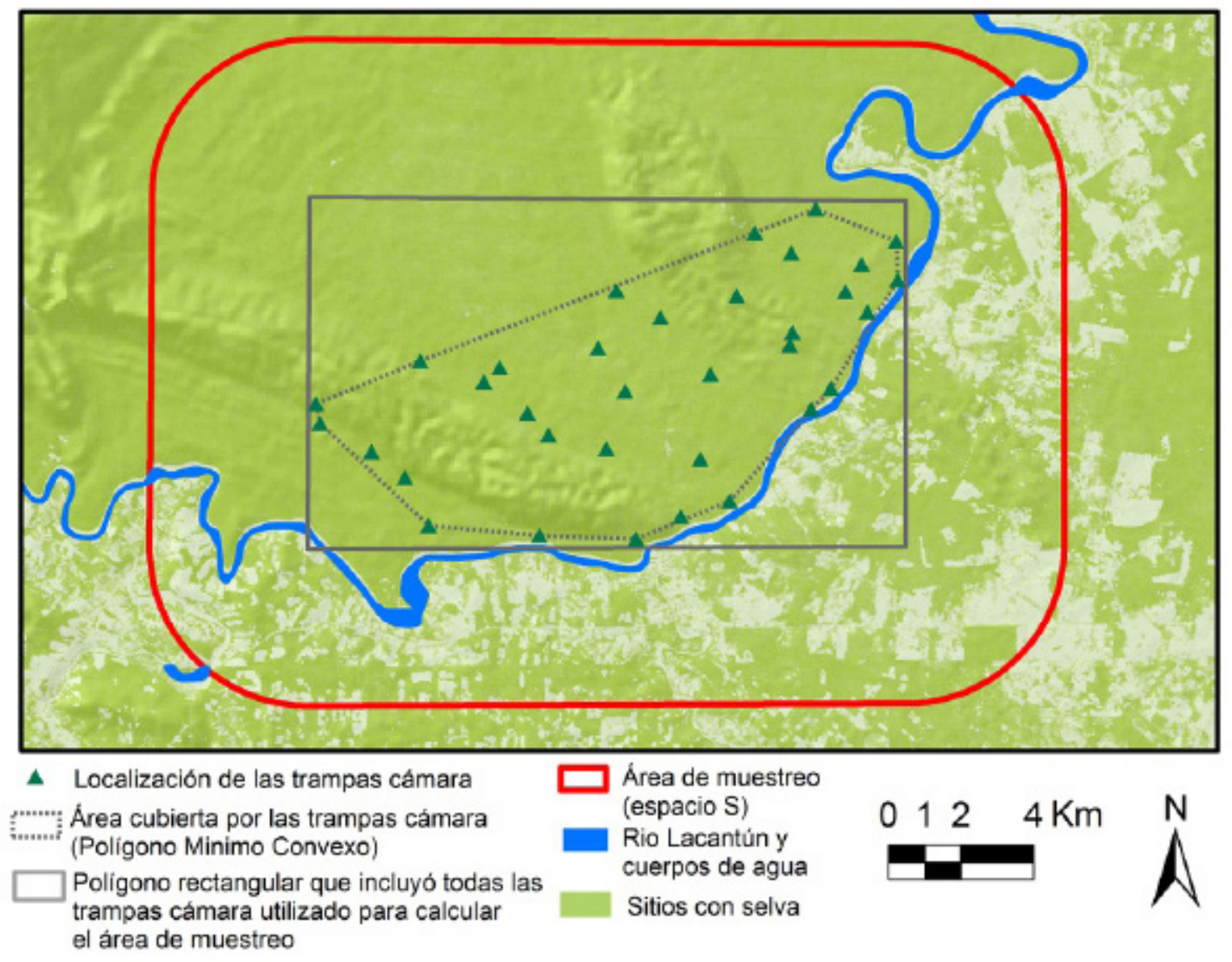

Figura 2. Localización de las trampas cámara y el área de muestreo. 
izquierdo y los fotografiados del perfil derecho. Debido a lo anterior, estimamos la densidad de la población utilizando los datos del perfil en el cual se obtuvieron más registros, por lo que las densidades estimadas en este estudio deben considerarse como las densidades mínimas para nuestra área de estudio.

Para la estimación de la densidad de los ocelotes utilizamos la técnica de captura-recaptura con trampas cámara y analizamos los datos a través de modelos espacialmente explícitos de captura-recaptura (SECR por sus siglas en inglés). Particularmente utilizamos los modelos bayesianos, los cuales utilizan métodos de Cadenas de Markov de Monte Carlo (MCMM por sus siglas en inglés) para el aumento de los datos, una técnica que complementa el conjunto de datos de los animales conocidos con un conjunto de datos arbitrarios derivados de todos las posibles historias de encuentro que consisten en ceros (Royle et al. 2009a, b). Una de las diferencias más importantes entre los modelos espacialmente explícitos y los modelos de captura-recaptura clásicos es que la unidad de estudio cambia. Para los primeros las unidades de estudio son los sitios de muestreo, es decir, las trampa -cámaras, para lo segundos, las unidades de estudio son los individuos de la especie. Lo anterior implica que a los modelos espacialmente explícitos se les puede incorporar información espacial sobre los movimientos y la ubicación de los individuos con respecto a la posición de las cámaras, lo que a su vez permite realizar estimaciones poblacionales tomando en cuenta la relación que existe entre la localización de las trampa cámaras y los centros de las áreas de actividad de los individuos fotografiados (Srivathsa et al. 2015). Estos modelos tienen el supuesto de que todos los individuos de la especie bajo estudio tienen un área de actividad relativamente definida y que los centros de éstas se distribuyen de manera uniforme en el área de interés (Royle et al. 2009a, b).

Para poder llevar a cabo los modelos espacialmente explícitos utilizamos el paquete SPACECAP (Gopalaswamy et al. 2012, 2015 disponible en https://cran.r-project.org/ web/packages/SPACECAP/index.html), para R 3.0.1 (2007; The R Foundation for Statistical Computing). Dado que la estimación de la densidad requiere la definición de un área de muestreo (conocido como espacio S) que incluya el área potencial que pueden utilizar los individuos en la periferia, calculamos un polígono rectangular que incluyó todas las estaciones de muestreo y agregamos un buffer de 4,352 metros, distancia que corresponde a la distancia máxima transitada por los ocelotes de acuerdo a las estaciones de muestreo (MMDM, por sus siglas en inglés; Figura 2). El tamaño de las celdas de la reja fue de $0.0625 \mathrm{~km}^{2}$ y cada punto para generar la reja se encontraba separado por $0.25 \mathrm{~km}$. Se realizaron 100,000 iteraciones con las simulaciones de las MCMC, con un periodo de descarga inicial de 5,000 y una tasa de adelgazamiento de 100. Establecimos que el número máximo de posibles individuos presentes fuera de casi 10 veces más (130) la cantidad de individuos realmente capturados. Para la evaluación de los resultados de los modelos nos basamos en los valores de Geweke. Para mayor detalle sobre el funcionamiento del paquete SPACECAP y de los modelos espacialmente explícitos de captura-recaptura se puede consultar Royle et al. (2009b), Gopalaswamy et al. (2012) y Gopalaswamy et al. (2015).

Análisis de los patrones de actividad. El patrón de actividad de los ocelotes se infirió por medio de la hora que registran las fotografías, debido a que las trampas-cámaras estuvieron activas durante todo el día. Se utilizaron todos los datos para la obtención de estos patrones, así como las fotografías que se obtuvieron de ambos sexos. Se agruparon las fotografías en intervalos de una hora durante las 24 horas del día. El 
análisis de los patrones de actividad se hizo utilizando el programa "Activity" (Rowcliffe $\underline{2015}$ - disponible en https://cran.r-project.org/web/packages/activity/index.html), para R 3.0.1 (2007; The R Foundation for Statistical Computing).

\section{Resultados}

Estimación de la densidad poblacional. Obtuvimos un total de 35 fotografías de ambos perfiles. Identificamos 14 individuos diferentes con el perfil derecho y 13 con el izquierdo (Tabla 1), por lo cual, utilizamos los primeros para llevar a cabo la estimación de la densidad poblacional. De éstos, fueron capturados cuatro machos y 10 hembras y se recapturaron a cuatro individuos diferentes. La frecuencia promedio de captura de los ocelotes fue de 18.2 capturas por 1,000 días trampa. El promedio de las distancias máximas viajadas por los ocelotes fue de 2,802 m (Tabla 1). Este promedio se basó solamente en las recapturas de dos individuos, ya que los otros dos individuos se recapturaron en los mismo sitios de su primera captura. El intervalo de las distancias máximas viajadas por los ocelotes fue de 1,252 a 4,352 m.

La densidad estimada fue de $12.9 \pm 5.39 \mathrm{SE} / 100 \mathrm{~km}^{2}$. Los valores de $\lambda 0$ (la tasa de detección que se espera si la trampa cámara se encontrara en el centro de actividad de los individuos) y $\psi$ (probabilidad de que uno de los valores aumentados que

Tabla 1. Número de individuos identificados por ambos perfiles.

\begin{tabular}{|c|c|c|c|c|c|c|c|}
\hline Lado & $\begin{array}{l}\text { Número de } \\
\text { capturas } \\
\text { independientes }\end{array}$ & $\begin{array}{l}\text { Número de } \\
\text { individuos } \\
\text { capturados }\end{array}$ & Machos & Hembras & $\begin{array}{c}\text { Sexo no } \\
\text { identificado }\end{array}$ & $\begin{array}{c}\text { Promedio de } \\
\text { las distancias } \\
\text { máximas que se } \\
\text { movieron (mts) }\end{array}$ & SD \\
\hline Derecho & 19 & 14 & 4 & 10 & - & 2,802 & 2,192 \\
\hline Izquierdo & 20 & 13 & 5 & 7 & 1 & 2,165 & 1,903 \\
\hline
\end{tabular}

representan individuos no capturados sea un individuo real) fueron de $1.51 \times 10^{-3} \pm$ $1.11 \times 10^{-3} \mathrm{SE}$ y $2.43 \times 10^{-1} \pm 1.05 \times 10^{-1} \mathrm{SE}$, respectivamente (Tabla 2). De acuerdo a los valores de Geweke obtenidos, todos los parámetros convergieron (Tabla 3).

Patrones de actividad. De acuerdo con el número de fotografías obtenidas, los ocelotes mostraron tres picos de actividad, tanto en el día como en la noche. El primer pico de actividad es en la madrugada y abarca desde las 01:00 a las 06:00 horas. El segundo es en la mañana y dura de las 10:00 a las 12:00 horas. El ultimo de las 21:00 a las 23:00 horas (Figura 3). La actividad de los ocelotes disminuye considerablemente entre las 08:00 y las 09:00 de la mañana y entre las 13:00 y las 16:00 de la tarde (Figura 3). Comparando los diferentes picos de actividad con los periodos de inactividad por

Tabla 2. Parámetros obtenidos en los modelos espacialmente explícitos de captura-recaptura

\begin{tabular}{lrr}
\hline & Valor estimado & Desviación Estándar \\
\hline $\mathbf{N}$ & $3.43 \times 10^{-1}$ & $1.43 \times 10^{-1}$ \\
Densidad* $^{*}$ & 0.129 & 0.0539 \\
$\lambda_{0}$ & $1.51 \times 10^{-3}$ & $1.11 \times 10^{-3}$ \\
$\sigma$ & $9.23 \times 10^{-3}$ & $1.51 \times 10^{-4}$ \\
$\psi$ & $2.43 \times 10^{-1}$ & $1.05 \times 10^{-1}$ \\
\hline
\end{tabular}

* Densidad estimada por SPACECAP en individuos por $\mathrm{km}^{2}$ 
medio del estadístico de Wald encontramos que el mayor pico de actividad es el de la madrugada. Los ocelotes fueron significativamente más activos en el pico de la madrugada que en los periodos de inactividad de la mañana ( $W=6.50 ; P<0.05$ ), y de la tarde $(\mathrm{W}=9.42 ; P<0.01)$. También este análisis reveló que los ocelotes son más activos en la madrugada que en el pico de actividad de la noche $(W=4.47 ; P<0.05)$. El análisis también reveló que los ocelotes son más activos en el pico de actividad de mañana (10:00 a las 12:00 horas) que en el periodo de inactividad de la tarde ( $\mathrm{W}=$ 3.96; $P<0.05$ ). Finalmente el porcentaje de tiempo activo de los ocelotes (nivel de actividad) fue de $0.53 \pm 0.08 \%(95 \% \mathrm{Cl}=0.31-0.63)$.

Figura 3. Frecuencia de actividad de los ocelotes obtenidos por medio de los registros de trampas cámara incluyendo los intervalos de confianza.

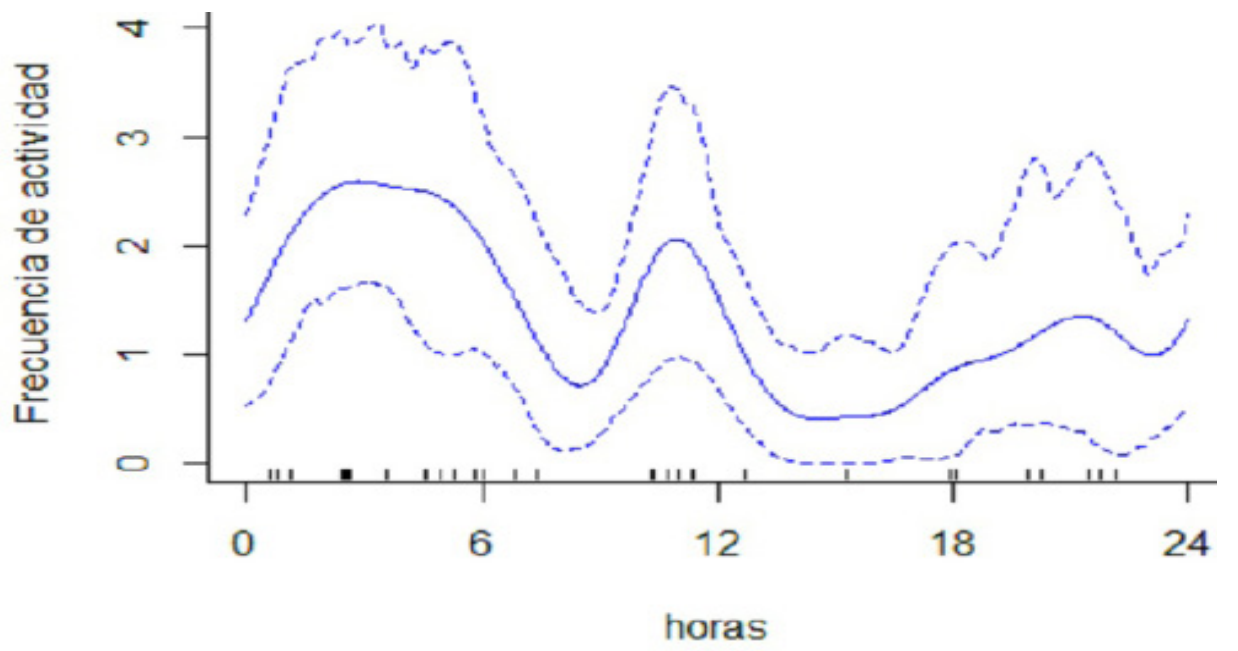

\section{Discusion}

De acuerdo a las densidades registradas de las poblaciones de ocelote en diversas partes de su distribución, la densidad reportada en este estudio (12.9 individuos/100 $\mathrm{km}^{2}$ ), se encuentra dentro del intervalo de densidad reportado en la literatura (Tabla 4). La densidad estimada para la Selva Lacandona es similar a la obtenida por otros autores utilizando los modelos clásicos de captura-recaptura y utilizando la distancia media máxima viajada por los ocelotes para calcular el área efectiva de muestreo. Dillon y Kelly (2007) reportaron una densidad de $25.89 \pm 7.92$ ocelotes $/ 100 \mathrm{~km}^{2}$ y de $12.61 \pm 3.66 / 100 \mathrm{~km}^{2}$ ocelotes utilizando los métodos de captura-recaptura clásicos y agregando un buffer de $1 / 2$ MMDM y MMDM, respectivamente, en Chiquibul en Belice. Asimismo, compararon los resultados con estimaciones obtenidas a partir de radio-collares para evaluar el desempeño de sus estimaciones y observaron que la distancia obtenida considerando $1 / 2$ MMDM representa menos de la mitad del radio de la distancia máxima registrada con radio collares; esto sugiere que para especies con ámbitos hogareños amplios como los ocelotes, la consideración de un buffer de MMDM completo podría ser más acertado, y que utilizar solamente el 1 1/2 MMDM podría ocasionar una sobreestimación de las densidades poblacionales (Soisalo y

Tabla 3. Valores de Geweke obtenidos para la validación del modelo.

\begin{tabular}{cccc}
\hline \multicolumn{1}{l}{ sigma } & lam0 & psi & N \\
\hline 0.2378 & -0.7647 & -1.0196 & -0.8378 \\
\hline
\end{tabular}


Cavalcanti 2005; Dillon y Kelly 2007). Bajo este contexto, consideramos que nuestra estimación mediante los modelos SECR es adecuada debido a que se encuentra más cercana a las estimaciones obtenidas por medio de los modelos captura-recaptura clásicos que utilizan el MMDM para estimar el área efectiva de muestreo obtenidas en ecosistemas similares (Tabla 4).

Las densidades de los ocelotes son muy variables a lo largo de su distribución (Tabla 4), y las diferencias entre las densidades estimadas en ecosistemas similares pueden deberse a problemas metodológicos como la distancia entre las trampas cámara, el esfuerzo de muestreo y el método utilizado para hacer la estimación, lo que dificulta las comparaciones entre los diferentes estudios (Dillon y Kelly 2007; 2008; MartínezHernández et al. 2015). Sin embargo, se ha observado que la densidad de los ocelotes a los largo del continente Americano aumenta conforme aumenta la precipitación y se reduce la distancia al ecuador (Di Bitetti et al. 2008). Por lo general las densidades más altas se encuentran en los ecosistemas tropicales más cercanos al ecuador (Tabla 4). Este patrón se explica debido a que la productividad primaria es mayor en los sitios más cercanos al ecuador, lo cual permite una mayor capacidad de carga de los ecosistemas y por lo tanto una mayor cantidad de presas disponibles para los depredadores debido a una mayor cantidad de presas disponibles (Di Bitetti et al. 2008; Kolowski y Alonso 2010). Por esta razón las densidades de los ocelotes en los

Tabla 4. Densidades de ocelotes estimadas a lo largo de su distribución. Se muestra el método que se utilizó para calcular el área efectiva de muestreo y el esfuerzo de muestreo. Número de estaciones (No. St)

\begin{tabular}{|c|c|c|c|c|c|c|}
\hline País & Área de estudio & $\begin{array}{l}\text { Método } \\
\text { utilizado }\end{array}$ & $\begin{array}{c}\text { Densidad } \\
\text { por } 100 \\
\text { km }^{2}\end{array}$ & $\begin{array}{l}\text { Noches- } \\
\text { trampa }\end{array}$ & No. St & Referencia \\
\hline EE. UU. & Valle del Río Grande & $1 / 2$ MMDM & 3 & 1,310 & 15 & Haines et al. (2006) \\
\hline \multirow[t]{4}{*}{ México } & Los Chimalapas & $1 / 2 \mathrm{MMDM}$ & 38 & 8,529 & 29 & Pérez-Irineo y Santos-Moreno (2014) \\
\hline & & MMDM & 22.6 & & & \\
\hline & Sierra Abra-Tanchipa & SECR & $3.0-18.0$ & 7,786 & $22-27$ & Martínez-Hernández et al. (2015) \\
\hline & Selva Lacandona & SECR & 12.9 & 1,920 & 33 & Este estudio \\
\hline \multirow[t]{2}{*}{ Belice } & Chiquibul & $1 / 2 \mathrm{MMDM}$ & 25.8 & 412 & 17 & Dillon y Kelly (2008) \\
\hline & & MMDM & 12.6 & & & \\
\hline Costa Rica & $\begin{array}{l}\text { Corredor Biológico } \\
\text { Talamanca Caribe }\end{array}$ & PMC & 6.4 & 600 & 10 & González-Maya y Cardenal-Porras (2011) \\
\hline \multirow[t]{2}{*}{ Panamá } & Isla de Barro Colorado & SECR & 15.87 & 1,824 & 21 & Rodgers et al. (2014) \\
\hline & & MMDM & 18.15 & & & \\
\hline \multirow[t]{2}{*}{ Colombia } & Palamarito & $1 / 2 \mathrm{MMDM}$ & 5.47 & 1,282 & 21 & Diaz-Pulido y Payán Garrido (2011) \\
\hline & & MMDM & 11.08 & & & \\
\hline \multirow[t]{4}{*}{ Ecuador } & Maxus Road (Yasuní) & $1 / 2$ MMDM & 83.5 & 2,340 & 27 & Salvador y Espinosa (2015) \\
\hline & & MMDM & 31.3 & & & \\
\hline & Lorocachi (Yasuní\}9 & $1 / 2$ MMDM & 93.3 & 2,340 & 27 & \\
\hline & & MMDM & 34.8 & & & \\
\hline \multirow[t]{2}{*}{ Perú } & Amazonas Peruano & $1 / 2$ MMDM & 94.7 & 2,085 & 23 & Kolowski y Alonso (2010) \\
\hline & & MMDM & 58.5 & & & \\
\hline Bolivia & Gran Chaco & MMDM & 19 & - & 15 & Maffei y Noss (2008) \\
\hline \multirow[t]{2}{*}{ Brasil } & Pantanal & $1 / 2$ MMDM & 56.4 & 450 & 6 & Trolle y Kéry (2003) \\
\hline & Caraguatá & $1 / 2$ MMDM & 4 & 4,250 & 42 & Goulart et al. (2009) \\
\hline \multirow[t]{2}{*}{ Argentina } & Urugua-i & $1 / 2$ MMDM & 13.3 & 1,409 & 34 & Di Bitetti et al. (2006) \\
\hline & & MMDM & 7.7 & & & \\
\hline
\end{tabular}


boques tropicales de Centroamérica, como en el caso de la Selva Lacandona, podrían ser menores (de tres a cinco veces) que las densidades reportadas en ecosistemas similares en Sudamérica próximos al ecuador.

Aunque la productividad primaria, la precipitación y la latitud aparentemente determinan la abundancia de los ocelotes a aparentemente determinan la abundancia de los ocelotes a una escala regional, factores como la fragmentación, cacería y la competencia con otras especies también afectan la abundancia de esta especie a escala local (Di Bitetti et al. 2008). Estudios con radio-telemetría de ocelotes en otras regiones tropicales han demostrado que esta especie puede adaptarse a utilizar ambientes modificados por el hombre. Incluso en muchos ambientes modificados los ocelotes son los depredadores tope después de que los felinos mayores, como el puma (Puma concolor) y el jaguar (Panthera onca), han sido extirpados (Cruz-Rodriguez et al. 2015). Otros estudios realizados en la región de la Amazonia han mostrado que tanto la densidad como la actividad de los ocelotes no se modifica con las actividades humanas (Kolowski y Alonso 2010; Salvador y Espinosa 2015). Entender como la cambia la abundancia de esta especie en distintos grados de fragmentación y perturbación humana debe ser una prioridad para diseñar los planes de conservación adecuados para esta especie en las regiones tropicales del sur de México.

Los ocelotes en la Selva Lacandona mostraron picos de actividad tanto en la noche como en el día. Varios estudios han reportado que la actividad de los ocelotes es principalmente nocturna (Maffei et al. 2005; Di Bitetti et al. 2010; Kolowski y Alonso 2010; Martínez-Hernández et al. 2015; Salvador y Espinosa 2015), o nocturna con un poco de actividad en el día (Di Bitetti et al. 2006; Pérez-Irineo y Santos-Moreno 2014; Briones-Salas et al. 2016). La actividad de los ocelotes está influenciada por los periodos de actividad de sus presas (Emmons 1987; Harmsen et al. 2011; Pérez-Irineo y SantosMoreno 2014; Martínez-Hernández et al. 2015; Salvador y Espinosa 2015). Los ocelotes son más activos en el día en sitios donde hay poca actividad humana y suelen limitar su actividad a la noche en sitios donde hay actividad humana y cacería (Kolowski y Alonso 2010; Pérez-Irineo y Santos-Moreno 2014). Por lo que el pico actividad diurno observado en la Selva Lacandona se puede explicar por la baja presencia humana en el área, debido a que la mayor parte de las trampas cámara estuvieron colocadas en áreas remotas con poca o nula actividad humana. Además existe evidencia de que los ocelotes pueden ajustar sus periodos de actividad para evitar la competencia por interferencia y la depredación intragremio de las demás especies de felinos con las cuales coexisten (Di Bitetti et al. 2010; Salvador y Espinosa 2015).

Los resultados de este estudio ilustran la importancia de la Selva Lacandona para conservar una población viable de ocelotes a largo plazo en México. La conservación de esta población y la de otras especies emblemáticas, depende de conservar las grandes extensiones de bosque tropical que aún se mantienen dentro de las áreas naturales protegidas de esta región (de la Torre y Medellín 2011).

\section{Agradecimientos}

Agradecemos a nuestros asistentes de campo por el apoyo a este proyecto, en especial a R. Lombera, I. Lombera, M. Cornelio, I. Díaz, H. Baldovinos y E. Baldovinos. Queremos también agradecer a todos los miembros del Laboratorio de Ecología y Conservación de Vertebrados Terrestres del Instituto de Ecología de la UNAM por el apoyo logístico y académico para llevar a cabo este proyecto en especial a R. A. 
Medellín, O. Gaona y A. Ibarra. También queremos agradecer a K. Tavera y G. Cerón quienes amablemente recopilaron información durante este proyecto. Agradecemos enormemente a Rufford Small Grants Foundation, Sea World \& Busch Garden Fund y Jaguar Conservation Program de WCS por el apoyo económico brindado. Finalmente agradecemos a la Comisión Nacional de Áreas Naturales Protegidas (CONANP) por todo el apoyo para realizar este estudio.

\section{Literatura citada}

Ahumada-Carrillo, I. T., J. C. Arenas-Monroy, y M. A. Íñiguez. 2013. Presence of the ocelot (Leopardus pardalis) in northern Jalisco, Mexico. Revista Mexicana de Biodiversidad 84:718-721.

Aranda, M., F. Botello, y L. López-De Buen. 2012. Diversidad y datos reproductivos de mamíferos medianos y grandes en el bosque mesófilo de montaña de la Reserva de la Biosfera Sierra de Manantlán, Jalisco-Colima, México. Revista Mexicana de Biodiversidad 83:778-784.

Bárcenas, H., y R. A. Medellín. 2010. Ocelot (Leopardus pardalis) in Aguascalientes, Mexico. Southwestern Naturalist 55:447-449.

Briones-Salas, M., I. Lira-Torres, R. Carrera-Treviño, y G. Sánchez-Rojas. 2016. Relative abundance and activity patterns of wild felids in Chimalapas rainforest, Oaxaca, Mexico. Therya 7:123-134.

Cruz-Rodriguez, C., J. F. Gonzalez-Maya, A. Rodriguez-Bolaños, A. A. Cepeda-Mercado, D. Zarrate-Charry, y J. R. Belant. 2015. Ocelot Leopardus pardalis (Carnivora: Felidae) in a fragmented landscape of Colombia. Revista Mexicana de Mastozoología 5:18-24.

Davis, S., V. Haywood, O. Herrera-Macbride, J. Villa-Lobos, y A. Hamilton. 1997. Centre of plants diversity. A guide and strategy for their conservation. The World Wide Fund for Nature, UICN and The World Conservation Union. Cambridge, Reino Unido.

De La Torre, J. A. 2009. Estimación poblacional del jaguar (Panthera onca) y abundancia relativa de sus presas en la Reserva de la Biosfera Montes Azules, Chiapas, México. Tesis de Maestría. Universidad Nacional Autónoma de México. Ciudad de México, México.

De La Torre, J. A., y R. A. Medellín. 2011. Jaguars Panthera onca in the greater Lacandona ecosystem, Chiapas, Mexico: population estimates and future prospects. Oryx 45:546-553.

De Villa, A., E. Martinez Meyer, y C. A. López-González. 1995. Ocelot (Leopardus pardalis) food habits in a tropical deciduous forest of Jalisco, Mexico. American Midland Naturalist 148:146-154.

Di Bitetti, M. S., C. D. De Angelo, Y. E. Di Blanco, y A. Paviolo. 2010. Niche partitioning and species coexistence in a Neotropical felid assemblage. Acta Oecologica 36:403-412.

Di Bitetti, M. S., A. Paviolo, y C. De Angelo. 2006. Density, habitat use and activity patterns of ocelots (Leopardus pardalis) in the Atlantic Forest of Misiones, Argentina. Journal of Zoology 270:153-163.

Di Bitetti, M. S., A. Paviolo, C. D. De Angelo, y Y. E. Di Blanco. 2008. Local and continental correlates of the abundance of a neotropical cat, the ocelot (Leopardus pardalis). Journal of Tropical Ecology 24:189-200.

Diaz-Pulido, A., y E. Payán Garrido. 2011. Densidad de ocelotes (Leopardus pardalis) en los Llanos Colombianos. Mastozoología Neotropical 18:63-71.

Dillon, A., Y M. J. Kelly. 2007. Ocelot Leopardus pardalis in Belize: the impact of trap spacing and distance moved on density estimates. Oryx 41:469-477. 
Dillon, A., y M. J. Kelly. 2008. Ocelot home range, overlap and density: comparing radio telemetry with camera trapping. Journal of Zoology 275:391-398.

Emmons, L. H. 1987. Comparative feeding ecology of felids in a neotropical rainforest. Behavioral Ecology and Sociobiology 20:271-283.

Galindo-Aguilar, R. E., L. Cacelin-Castillo, O. C. Rosas-Rosas, M. G. Bravo-Vinaja, J. L. Alcántara-Carbajal, y V. Vázquez-García. 2016. First records of ocelot in tropical forests of the Sierra Negra of Puebla and Sierra Mazateca de Oaxaca, Mexico. Therya 7:205-211.

García-Bastida, M., F. Martínez-De la Fuente, A. Váquez-Venegas, y J. L. Peña-Mondragón. 2016. A new record of ocelot in Parque Ecológico. Therya 7:1-6.

González-Maya, J. F., y J. Cardenal-Porras. 2011. Ocelot density in the Caribbean slope of the Talamanca region, Costa Rica. Hystrix 22:355-360.

Gopalaswamy, A. M., A. J. Royle, M. E. Meredith, P. Singh, S. Jathanna, Devcharan N. Kumar, y K. U. KARANTH. 2015. SPACECAP - A Program to estimate animal abundance and density using bayesian spatially-explicit capture-recapture models. <https://cran.r-project. org/web/packages/SPACECAP/index.html>

Gopalaswamy, A. M., J. A. Royle, J. E. Hines, P. Singh, D. Jathanna, N. S. Kumar, y K. U. Karanth. 2012. Program SPACECAP: Software for estimating animal density using spatially explicit capture-recapture models. Methods in Ecology and Evolution 3:1067-1072.

Goulart, F., M. E. Graipel, M. Tortato, I. Ghizoni-Jr, L. G. Oliveira-Santos, y N. Cáceres. 2009. Ecology of the ocelot (Leopardus pardalis) in the Atlantic Forest of Southern Brazil. Neotropical Biology and Conservation 4:137-143.

Grigione, M., A. Caso, R. List, y C. López-Gonzalez. 2001. Felid conservation status and conservation of endangered cats along the U.S.-Mexico border. Endangered Species UPDATE 18:129-132.

Haines, A. M., J. E. Janecka, M. E. Tewes, L. I. Grassman JR., y P. Morton. 2006. The importance of private lands for ocelot Leopardus pardalis conservation in the United States. Oryx 40:90-94.

HaLL, E. 1981. The Mammals of North America. Volumen I. John Wiley and Sons. New York, EE. UU.

Harmsen, B. J., R. J. Foster, S. C. Silver, L. E. T. Ostro, y C. P. Doncaster. 2011. Jaguar and puma activity patterns in relation to their main prey. Mammalian Biology 76:320-324.

Hernández-Flores, S. D., G. Vargas-Licona, y G. Sánchez-Rojas. 2013. First records of the Ocelot (Leopardus pardalis) in the state of Hidalgo, Mexico. Therya 4:99-102.

Iglesias, J., V. Sánchez-Cordero, G. Magaña-Cota, R. Bolaños, M. Aranda, R. Hernández, y F. J. Botello. 2008. Noteworthy records of margay, Leopardus wiedii and ocelot, Leopardus pardalis in the state of Guanajuato, Mexico. Mammalia 72:347-349.

INE-SEMARNAP. 2000. Programa de manejo Reserva de la Biosfera Montes Azules. Dirección Ejecutiva de Participación Social, Enlace y Comunicación, Instituto Nacional de Ecología. Ciudad de México, México.

Jong, B. H. J. De, S. Ochoa-Gaona, y M. A. Castillo-Santiago. 2000. Carbon flux and patterns of land-use / land-cover change in the Selva Lacandona. Ambio 29:504-511.

Kolowskı, J. M., y A. Alonso. 2010. Density and activity patterns of ocelots (Leopardus pardalis) in northern Peru and the impact of oil exploration activities. Biological Conservation 143:917-925.

Leopold, A. S. 1959. Fauna Silvestre de México. Editorial Pax. Ciudad de México, México.

Lorenzana, P. G., G. R. A. Castillo, y C. A. López-González. 2004. Distribution, habitat association, and activity patterns of medium and large-sized mammals of Sonora, Mexico. Natural Areas Journal 24:352-357. 
Maffel, L., Y A. J. Noss. 2008. How small is too small ? Camera trap survey areas and density estimates for ocelots in the Bolivian Chaco. Biostropica 40:71-75.

Maffei, L., A. J. Noss, E. Cuellar, y D. I. Rumiz. 2005. Ocelot (Felis pardalis) population densities, activity, and ranging behaviour in the dry forests of eastern Bolivia: data from camera trapping. Journal of Tropical Ecology 21:1-6.

Martínez-Calderas, J. M., O. C. Rosas-Rosas, J. F. Martínez-Montoya, L. A. Tarango-Arámbula, F. Clemente-Sánchez, M. M. Crosby-Galván, y M. D. Sánchez Hermosillo. 2011. Distribución del ocelote (Leopardus pardalis) en San Luis Potosí, México. Revista mexicana de biodiversidad 82:997-1004.

Martínez-Hernández, A., O. C. Rosas-Rosas, F. Clemente-Sánchez, L. A. Tarango-Arámbula, J. Palacio-Núnez, L. C. Bender, y J. G. Herrera-Haro. 2015. Density of threatened ocelot Leopardus pardalis in the Sierra Abra-Tanchipa Biosphere Reserve, San Luis Potosí, Mexico. Oryx 49:619-625.

Medellín, R. A. 1994. Mammals diversity and conservation in the Selva Lacandona, Chiapas, Mexico. Conservation Biology 8:780-799.

Mendoza, E., Y R. Dirzo. 1999. Deforestation in Lacandonia (southeast Mexico): evidence for the declaration of the northernmost tropical hot-spot. Biodiversity and Conservation 8:1621-1641.

NowelL, K., Y P. Jackson. 1996. Wild Cats Status, survey and conservation action plan. IUCN/ SSC Cats Specialist Group. The World Conservation Union. Gland, Suiza.

O'BRIEN, K. L. 1998. Tropical deforestation and climate change: What does the record reveal? The Professional Geographer 50:140-153.

Paviolo, A., P. Crawshaw, A. Caso, T. De Oliveira, C.A. López-González, M. Kelly, C. De Angelo, y E. Payán. 2015. Leopardus pardalis. The IUCN Red List of Threatened Species 2015: e.T11509A50653476 <http://dx.doi.org/10.2305/IUCN.UK.2015-4. RLTS.11509A50653476.en.>

Pérez-Irineo, G., y A. Santos-Moreno. 2014. Density, distribution, and activity of the ocelot Leopardus pardalis (Carnivora: Felidae) in Southeast Mexican rainforests. Revista de Biología Tropical 62:1421-1432.

Pozo De La Tijera, C., y J. E. Escobedo Cabrera. 1999. Mamíferos terrestres de la Reserva de la Biosfera de Sian Ka an, Quintana Roo, México. Revista de Biología Tropical 47:251-262.

Ramírez-Bravo, O. E., E. Bravo-Carrete, C. Hernández-Santín, S. Schinkel-Brault, y K. Chris. 2010. Ocelot (Leopardus pardalis) distribution in the state of Puebla , Central Mexico. Therya 1:111-120.

Rodgers, T. W., J. Giacalone, E. J. Heske, J. E. Janečka, C. A Phillips, y R. L. Schooley. 2014. Comparison of noninvasive genetics and camera trapping for estimating population density of ocelots (Leopardus pardalis) on Barro Colorado Island, Panama. Tropical Conservation Science 777:690-705.

RowclifFe, M. 2015. Package "activity" - Animal Activity Statistics. <https://cran.r-project. org/web/packages/activity/index.html>.

Royle, J. A., K. U. Karanth, A. M. Gopalaswamy, y N. S. Kumar. 2009a. Bayesian inference in camera trapping studies for a class of spatial capture-recapture models. Ecology 90:3233-3244.

Royle, J. A., J. D. Nichols, K. U. Karanth, y A. M. Gopalaswamy. 2009b. A hierarchical model for estimating density in camera-trap studies. Journal of Applied Ecology 46:118-127.

SALVADOR, J., Y S. EsPinOSA. 2015. Density and activity patterns of ocelot populations in Yasuní National Park, Ecuador. Mammalia. 79: DOI: 10.1515/mammalia-2014-0172

Sánchez Hernández, C., J. A. Almazán-Catalán, F. Ruiz-Gutiérrez, M. D. L. Romero-Almaraz, A. Taboada-Salgado, E. Beltrán-Sánchez, y L. Sánchez-Vázquez. 2013. Registros adicionales de felinos del estado de Guerrero, México. Revista Mexicana de Biodiversidad 84:347-359. 
Sanchez, O., J. Ramirez-Pulido, U. Aguilera-Reyes, y O. Monroy-Vilchis. 2002. Felid record from the State of Mexico, Mexico. Mammalia 66:289-294.

SEMARNAT. 2010. Norma Oficial Mexicana. NOM-059-SEMARNAT-2010. Diario Oficial de la Federación.

Servín, J., E. Chacón, N. Alonso-Pérez, y C. Huxley. 2003. New records of mammals from Durango, Mexico. The Southwestern Naturalist 48:136-138.

Solsalo, M. K., y S. M. C. Cavalcanti. 2005. Estimating the density of a jaguar population in the Brazilian Pantanal using camera-traps and capture - recapture sampling in combination with GPS radio-telemetry. Biological Conservation 129:487-496.

Srivathsa, A., R. Parameshwaran, S. Sharma, y K. U. Karanth. 2015. Estimating population sizes of leopard cats in the Western Ghats using camera surveys. Journal of Mammalogy 96:742-750.

Sunquist, M. E., y F. C. Sunquist. 2009. Family Felidae. in Handbook of the mammals of the world, Vol. 1, Carnivores (Wilson, D. E., y R. A. Mittermeier, eds.). Lynx Edicions. Barcelona, España.

Trolle, M., Y M. Kéry. 2003. Estimation of ocelot density in the pantanal using capturerecapture analysis of camera-trapping data. Journal of Mammalogy 84:607-614.

Trolle, M., Y M. Kéry. 2005. Camera-trap study of ocelot and other secretive mammals in the northern Pantanal. Mammalia 69:2-9.

Valdez-Jiménez, D., C. M. García-Balderas, y G. E. Quintero-Díaz. 2013. Presencia del ocelote (Leopardus pardalis) en la "Sierra del Laurel", municipio de Calvillo, Aguascalientes, México. Acta Zoológica Mexicana 29:688-692.

Submited: January 16, 2016

Reviewed: April 1, 2016

Accepted: April 26, 2016

Associated editor: Lía Mendez 
DENSIDAD DE OCELOTES EN LA SELVA LACANDONA

270 THERYA Vol. 7 (2): 257-269 